\title{
Periodontal Ligament Stem Cells in the Periodontitis Microenvironment Are Sensitive to Static Mechanical Strain
}

\author{
Jia Liu, ${ }^{1}$ Qiang Li, ${ }^{2}$ Shiyu Liu, ${ }^{3}$ Jie Gao, ${ }^{1}$ Wen Qin, ${ }^{1}$ Yang Song, ${ }^{4}$ and Zuolin Jin ${ }^{1}$ \\ ${ }^{1}$ State Key Laboratory of Military Stomatology and National Clinical Research Center for Oral Diseases and \\ Shaanxi Clinical Research Center for Oral Diseases, Department of Orthodontics, School of Stomatology, \\ The Fourth Military Medical University, Xian, Shaanxi 710032, China \\ ${ }^{2}$ State Key Laboratory of Military Stomatology and National Clinical Research Center for Oral Diseases and \\ Shaanxi International Joint Research Center for Oral Diseases, Department of General Dentistry \& Emergency, \\ School of Stomatology, The Fourth Military Medical University, Xian, Shaanxi 710032, China \\ ${ }^{3}$ State Key Laboratory of Military Stomatology and National Clinical Research Center for Oral Diseases and \\ Shaanxi International Joint Research Center for Oral Diseases, Department of Oral Histology and Pathology, \\ School of Stomatology, The Fourth Military Medical University, Xian, Shaanxi 710032, China \\ ${ }^{4}$ Department of Stomatology, No. 323 Hospital of PLA, Xian, Shaanxi 710054, China
}

Correspondence should be addressed to Qiang Li; lqaq726@163.com and Zuolin Jin; zuolinj@fmmu.edu.cn

Received 6 October 2016; Accepted 16 January 2017; Published 21 February 2017

Academic Editor: Tong-Chuan He

Copyright (C) 2017 Jia Liu et al. This is an open access article distributed under the Creative Commons Attribution License, which permits unrestricted use, distribution, and reproduction in any medium, provided the original work is properly cited.

During orthodontic treatment, periodontium remodeling of periodontitis patients under mechanical force was abnormal. We have previously confirmed the function impairment of periodontal ligament stem cells (PDLSCs) in the periodontitis microenvironment which might be involved in this pathological process. However, the response of PDLSCs in periodontitis microenvironment to mechanical force remains unclear. Therefore, in the present study, we introduced a Flexcell tension apparatus and investigated the response of PDLSCs obtained from periodontal tissues of periodontitis patients (PPDLSCs) and of those obtained from healthy periodontal tissues (HPDLSCs) to different magnitudes of static mechanical strain (SMS). PPDLSCs showed increased proliferation, decreased osteogenic activity, activated osteoclastogenesis, and greater secretion of inflammatory cytokines. Different magnitudes of SMS exerted distinct effects on HPDLSCs and PPDLSCs. An SMS of 12\% induced optimal effects in HPDLSCs, including the highest proliferation, the best osteogenic ability, the lowest osteoclastogenesis, and the lowest secretion of inflammatory cytokines, while the optimal SMS for PPDLSCs was 8\%. Excessive SMS damaged PPDLSCs function, including decreased proliferation, an imbalance between osteogenesis and osteoclastogenesis, and an activated inflammatory response. Our data suggest that PPDLSCs are more sensitive and less tolerant to SMS, and this may explain why mechanical force results in undesirable effects in periodontitis patients.

\section{Introduction}

During orthodontic tooth movement, appropriate orthodontic force can activate biological responses in periodontal tissues [1], including bone formation on the tension side, bone resorption on the compression side, and reattachment of the periodontal ligament (PDL) [2]. In this intricate biological process, the PDL plays a crucial role in maintaining periodontal tissue homeostasis to prevent undesired pathologic conditions [3]. However, currently, orthodontic treatments are no longer confined to adolescents, most of whom have a healthy periodontium. In contrast, an increasing number of adults are attending orthodontic clinics to obtain a charming smile and stomatognathic health, and most of these adults present with mild or severe periodontal disease [4]. Periodontitis with destruction of periodontal tissue and alveolar bone results in increased production of several osteoclastogenic cytokines, such as IL-6, IL-8, IL-1 $\beta$, 
and TNF- $\alpha[5,6]$. These cytokines contribute to further periodontal damage. In the absence of inflammatory control, orthodontic treatments could easily lead to rapid loss of periodontal attachment and alveolar bone resorption [7]. Even after the completion of basic periodontal treatment, the regeneration and remodeling capacities of periodontal tissues appear to be decreased in patients with periodontitis $[8,9]$.

At the cellular level, orthodontic force leads to functional changes in cells in the periodontium. For example, the cell membrane, cytoskeleton, and nuclear protein matrix and genome exhibit functional changes [10]. A considerable amount of evidence has confirmed the presence of adult mesenchymal stem cells (MSCs) in periodontal tissues that maintain tissue homeostasis and regenerative capacity [8]. Periodontal ligament stem cells (PDLSCs) are one of the predominant types of MSCs involved in periodontal tissue regeneration because they not only regenerate cementumand PDL-like tissues in vivo [11] but also show better organization homology in terms of morphology, structure, and other organizational characteristics $[12,13]$. As PDLSCs are load sensitive, studies have shown that mechanical stimulation at the proper strength and frequency promotes the proliferation and osteogenic differentiation of PDLSCs [14]. Moreover, when the PDL is exposed to orthodontic-related mechanical forces, the tissues are reconstructed to balance osteogenesis and osteoclastogenesis. During this process, PDLSCs play a key role in bone formation, while RANKL provides a crucial signal for osteoclast formation [15]. Alveolar bone resorption and periodontal attachment loss occur if the balance between osteogenesis and osteoclastogenesis is disturbed by unsuitable mechanical forces.

Our group previously confirmed that the biological characteristics of PDLSCs are affected by extracellular microenvironment such as inflammation $[9,16]$ and aging $[17,18]$. In the periodontitis microenvironment, the proliferation capability of PDLSCs obtained from patients diagnosed with periodontitis (PPDLSCs) is increased, but the osteogenic and adipogenic potentials are decreased, which induces unfavorable periodontal regeneration. Given that an inflammatory microenvironment can impair stem cell properties, it is reasonable to hypothesize that PPDLSCs respond differently to mechanical forces compared with PDLSCs obtained from healthy periodontal tissues (HPDLSCs), which may lead to elevated osteoclastic activity and alveolar bone resorption in cases of periodontitis. In this study, we evaluated the response of PPDLSCs and HPDLSCs to SMS and investigated the best SMS magnitude for each cell population.

\section{Materials and Methods}

2.1. Enrollment of Subjects and Ethics Statement. HPDLSCs for primary cultures were obtained from 10 orthodontic patients $(37.9 \pm 7.2$ years old) undergoing premolar and third molar extractions with healthy periodontal tissue. PPDLSCs for primary cultures were obtained from 8 orthodontic patients $(38.9 \pm 7.9$ years old $)$ who were diagnosed with moderate chronic periodontitis by the same periodontics specialist. The diagnosis was based on the clinical symptoms of positive bleeding on probing, periodontal pocket depth $\leq 6 \mathrm{~mm}$, attachment loss of $3 \sim 4 \mathrm{~mm}$, and X-ray images of horizontal alveolar bone resorption by $1 / 3 \sim 1 / 2$ of the root length. We extracted the teeth from the periodontitis patients for orthodontic reasons and harvested the PPDLSCs. To ensure that the biological characteristics of the PDLSCs were not affected by factors other than inflammation, no medicine was used for periodontal treatment. None of the patients in this study had a history of systemic disease, smoking, or special medication. All the samples were collected at the Department of Oral and Maxillofacial Surgery, School of Stomatology, Fourth Military Medical University. All the participants provided written informed consent. The study was carried out in accordance with The Code of Ethics of the World Medical Association (Declaration of Helsinki), and it is approved by the Ethics Committee of the School of Stomatology, Fourth Military Medical University (Xi'an, China).

2.2. Cell Culture. The primary cells were cultured, and homogeneous populations of HPDLSCs and PPDLSCs were obtained from single cell-derived colonies by the limiting dilution technique as previously described $[9,11,16]$. These colonies were mixed to obtain multiple colony-derived PDLSCs. Then, stem cells from different individuals were pooled for subsequent use. All the primary cells used in this study were at passage 3 .

2.3. SMS Loading. For SMS loading, HPDLSCs or PPDLSCs were seeded onto collagen I-coated 6-well Bioflex plates (Flexcell International, Burlington, NC, USA) at a density of $3 \times 10^{5}$ cells/well. Cells achieving 95\% confluence were serum starved in $\alpha$ MEM for $24 \mathrm{~h}$ and then subjected to SMS using a Flexcell Tension Plus system (FX-4000T, Flexcell International, Burlington, NC, USA) capable of producing different strain magnitudes, including $6 \%, 8 \%, 10 \%, 12 \%$, and $14 \%$ elongation, at a frequency of $0.1 \mathrm{~Hz}$ [19]. The HPDLSCs and PPDLSCs control groups were also seeded onto Bioflex plates but were not subjected to strain. After $12 \mathrm{~h}$ of loading or stationary culture, the HPDLSCs or PPDLSCs were used for further experiments.

2.4. Flow Cytometric Analysis. Approximately $5 \times 10^{5}$ HPDLSCs or PPDLSCs were incubated with PE-conjugated monoclonal antibodies against human Stro-1, CD146, CD90, CD29, CD31, or CD14 (BD Biosciences, San Jose, CA, USA) for phenotypic identification of PDLSCs. The cells were subjected to flow cytometric analysis using a Beckman Coulter Epics XL instrument (Beckman Coulter, Fullerton, CA, USA) after incubation with specific antibodies as described previously [16].

For cell cycle analysis, HPDLSCs and PPDLSCs (approximately $2 \times 10^{4}$ cells) were cultured in 6-well plates with serum-free $\alpha \mathrm{MEM}$ for $24 \mathrm{~h}$ and exposed to different magnitudes of SMS for $12 \mathrm{~h}$. Then, HPDLSCs or PPDLSCs were prepared and exposed to trypsin/EDTA for $5 \mathrm{~min}$. Cell precipitates were washed twice with $0.01 \mathrm{M}$ PBS and resuspended in $1 \mathrm{~mL}$ physiologic saline by repeated vibration to ensure a single cell suspension. Then, $2 \mathrm{~mL}$ of cold dehydrated 
TABle 1: Primer sequences.

\begin{tabular}{|c|c|}
\hline Gene & Primer sequence \\
\hline & Forward $5^{\prime}$-TGG CAC CCA GCA CAA TGA A-3' \\
\hline$\beta$-Actin & $\begin{array}{l}\text { Reverse } 5^{\prime} \text {-CTA AGT CAT AGT CCG CCT AGA } \\
\text { AGC A-3 } 3^{\prime}\end{array}$ \\
\hline \multirow{2}{*}{ Runx2 } & Forward 5'-CCC GTG GCC TTC AAG GT-3' \\
\hline & Reverse $5^{\prime}$-CGT TAC CCG CCA TGA CAG TA-3' \\
\hline \multirow{2}{*}{ ALP } & Forward 5'-GGA CCA TTC CCA CGT CTT CAC-3' \\
\hline & Reverse $5^{\prime}$-CCT TGT AGC CAG GCC CAT TG-3' \\
\hline \multirow{2}{*}{ OPG } & Forward 5'-TTGAAATGGCAGTTGATTCCTTT-3' \\
\hline & Reverse $5^{\prime}$-TATCCTCTTTCTCAGGGTGCTTG-3' \\
\hline \multirow{2}{*}{ RANKL } & Forward 5'-ACCGACATCCCATCTGGTT- $3^{\prime}$ \\
\hline & Reverse 5'-GCCATCCTGATTAACTATTAGTT-3' \\
\hline \multirow{2}{*}{ C-fos } & Forward 5'-GACAGCCTTTCCTACTACCATTCC-3' \\
\hline & Reverse 5'-CGCAAAAGTCCTGTGTGTTGA-3' \\
\hline
\end{tabular}

alcohol was mixed quickly with the cell suspension to fix cells at $-4^{\circ} \mathrm{C}$ for $24-48 \mathrm{~h}$. Finally, the cells were washed twice again with PBS, stained with propidium iodide $(100 \mathrm{mg} / \mathrm{mL}$; Sigma, St. Louis, MO) at $48 \mathrm{C}$ for $30 \mathrm{~min}$, and subjected to flow cytometry (Beckman Coulter). One million cells were counted per sample. The fractions of cells in the G1, S, and G2 phases of the cell cycle were measured, and the proliferation index (PI, the percentage of cells in G2 + S) was determined.

2.5. Cell Viability Assays. After SMS loading, HPDLSCs or PPDLSCs were plated at a density of $2 \times 10^{3}$ cells/well in 96well plates and cultured in basal medium. After 5 days, a 3(4,5-dimethylthiazol-2-yl)-2,5-diphenyltetrazolium bromide (MTT) assay (Beyotime, China) was performed as previously described [20]. Briefly, $20 \mu \mathrm{L}$ of MTT agentia was added and the plates were incubated for $4 \mathrm{~h}$ at $37^{\circ} \mathrm{C}$. Then, $150 \mu \mathrm{L}$ of dimethyl sulfoxide (DMSO) was added to each well and the plates were incubated for $10 \mathrm{~min}$ at room temperature. Subsequently, the absorbance was measured at $490 \mathrm{~nm}$ using the plate reader (Sunrise Remote, Tecan).

2.6. Real-Time RT-PCR. After SMS loading, total RNA was isolated from HPDLSCs and PPDLSCs (TRIzol; Invitrogen) and converted to cDNA (Super Script First-Strand Synthesis Kit; Invitrogen). Real-time RT-PCR was performed using a QuantiTect SYBR Green PCR Kit (Toyobo, Osaka, Japan) and an Applied Biosystems 7500 Real-Time PCR Detection System. The primers for osteogenic (Runx2, ALP, and OPG) and osteoclastic (RANKL and C-fos) genes are listed in Table 1. Each reaction was performed in triplicate.

2.7. Osteogenic and Adipogenic Differentiation Assays. For osteogenesis assays, HPDLSCs and PPDLSCs were exposed to different magnitudes of SMS for $12 \mathrm{~h}$, and after reaching $80 \%$ confluence, HPDLSCs and PPDLSCs were cultured in osteogenic medium for 7-21 days and prepared for the differentiation analysis as previously described [21]. Alkaline phosphatase (ALP) staining was performed using the BCIP/NBT Alkaline Phosphatase Color Development Kit (Beyotime,
Shanghai, China) at day 7, and ALP activity was determined using an Alkaline Phosphatase (AKP/ALP) Detection Kit (Jiancheng Bioengineering, Nanjing, China) at day 7. Mineralized nodules were stained with Alizarin Red S (pH 4.2) (Kermel, Tianjin, China) for $15 \mathrm{~min}$ at room temperature at day 21 , and calcium levels were measured quantitatively using a calcium colorimetric assay kit (BioVision, San Francisco, CA, USA). For adipogenic assays, HPDLSCs and PPDLSCs were plated at a density of $5 \times 10^{4}$ cells per well in 6 -well plates. After reaching $80 \%$ confluence, the HPDLSCs/PPDLSCs were cultured in adipogenic medium $(\alpha \mathrm{MEM})$ supplemented with 5\% FBS, $0.5 \mathrm{mM}$ methylisobutylxanthine (Sigma, Santa Clara, CA, USA), $0.5 \mu$ M hydrocortisone (Sigma, Santa Clara, CA, USA), and $60 \mu \mathrm{M}$ indomethacin (Sigma, Santa Clara, CA, USA) for 7-21 days. For analysis, the adipogenic cultures were fixed in $4 \%$ paraformaldehyde for $30 \mathrm{~min}$ and stained with fresh Oil Red O solution (Sigma, Santa Clara, CA, USA) for $15 \mathrm{~min}$. To quantify the amount of Oil Red O-stained lipids, the stain was solubilized in isopropanol for 5 minutes at room temperature. The solubilized stain $(150 \mu \mathrm{L})$ was then transferred to the wells of a 96-well plate, and the absorbance was measured at $520 \mathrm{~nm}$.

2.8. ELISA Assays. HPDLSCs and PPDLSCs were exposed to different magnitudes of SMS loading for $12 \mathrm{~h}$ and cultured for another $24 \mathrm{~h}$. Then, the concentrations of IL- 6 , IL-8, IL-1 $\beta$, and TNF- $\alpha$ in supernatants from HPDLSCs and PPDLSCs cultures were determined using ELISA kits (Westang, Shanghai, China) according to the manufacturer's instructions.

2.9. Statistical Analysis. Statistical analyses were performed using the SPSS 16.0 software package (SPSS Inc., USA). Data acquisition and analyses were performed in a blinded manner. Significant differences in each measure were determined by one-way analysis of variance (ANOVA) and the Bonferroni post hoc test. Additionally, Student's $t$-test was used to analyze the HPDLSCs and PPDLSCs control groups. Statistical significance was set at $P<0.05$. Data are presented as the mean \pm standard deviation. Each experiment was performed three times.

\section{Results}

3.1. Culture and Identification of HPDLSCs and PPDLSCs. Primary cultures were successfully obtained, and putative HPDLSCs (Figure 1(a)) and PPDLSCs (Figure 1(b)) were isolated. Using a microscope, HPDLSCs and PPDLSCs were observed to grow in an adherent manner with a long spindle shape, and there were no obvious differences between the two cell populations. Both HPDLSCs and PPDLSCs expressed the MSC markers Stro-1, CD146, CD90, and CD29 and were negative for the hematopoietic markers CD31 and CD14 (Figures 1(c) and 1(d)). Compared with PPDLSCs, HPDLSCs showed stronger expression of Stro-1, CD146, CD90, and CD29 $(P<0.05)$. However, there were no significant differences in CD31 and CD14 expression between the two types of PDLSCs $(P>0.05)$ (Figure 1(e)). These data indicated that PPDLSCs from an inflammatory microenvironment more weakly expressed MSC markers compared with HPDLSCs 
HPDLSCs

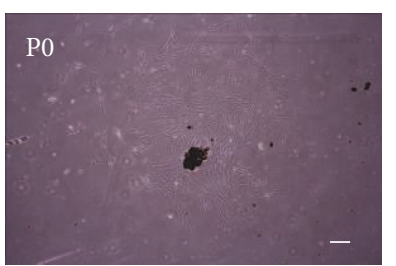

(a)

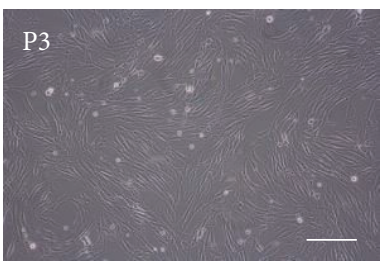

Cont: $1.4 \%$

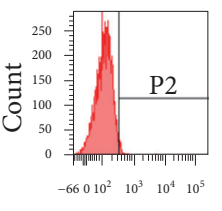

FITC-A

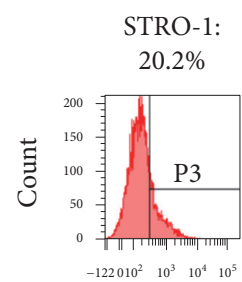

PE-A

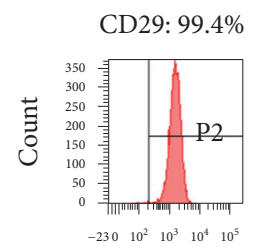

FITC-A
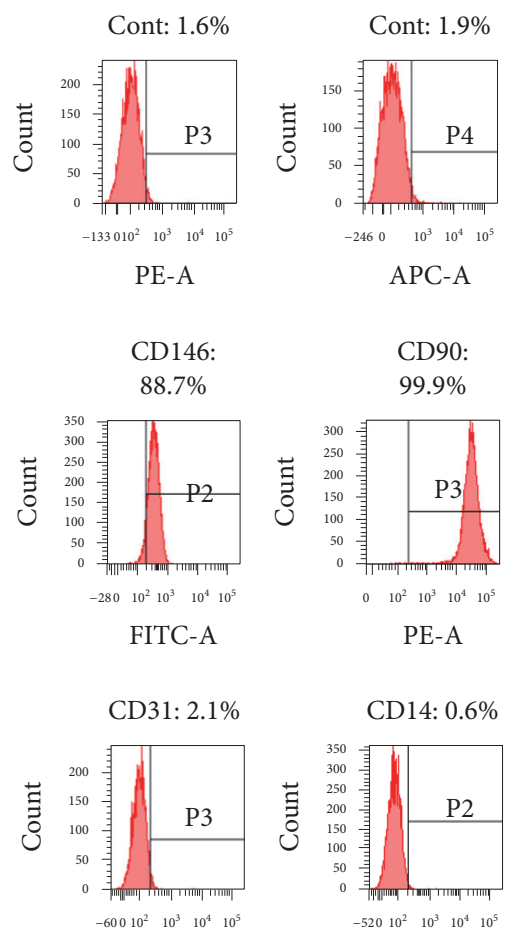

PE-A

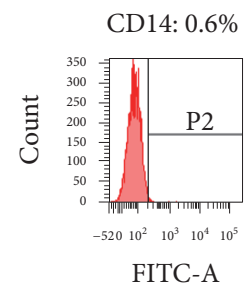

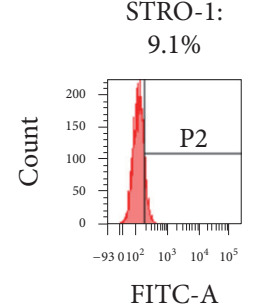

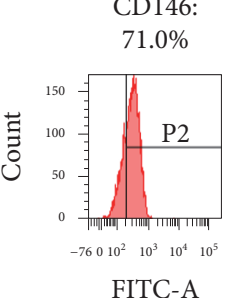

(b)
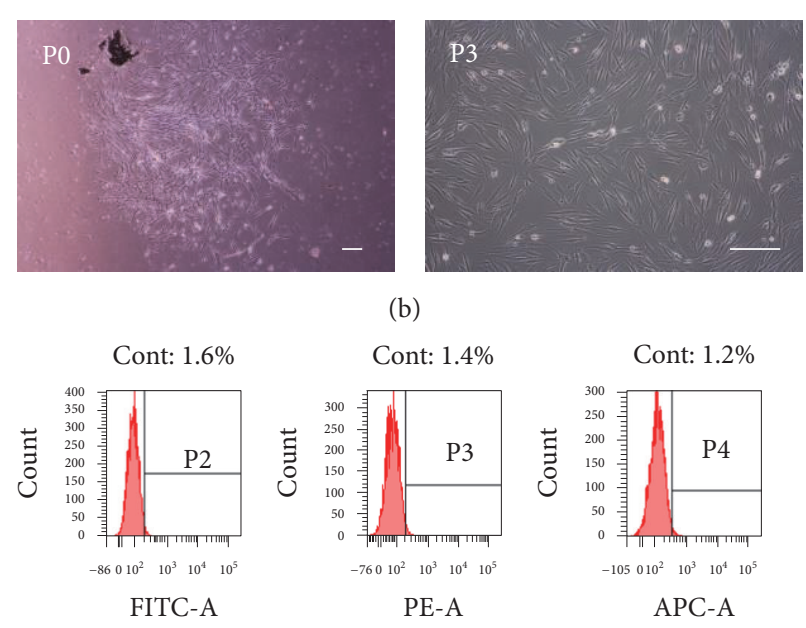

(c)
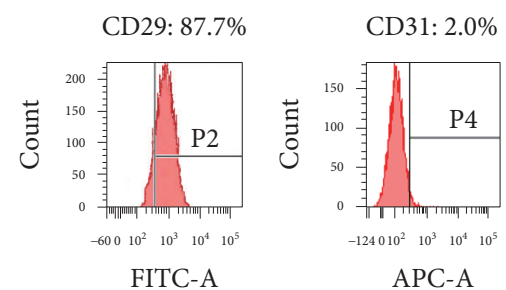

APC-A

(d)

HPDLSCs
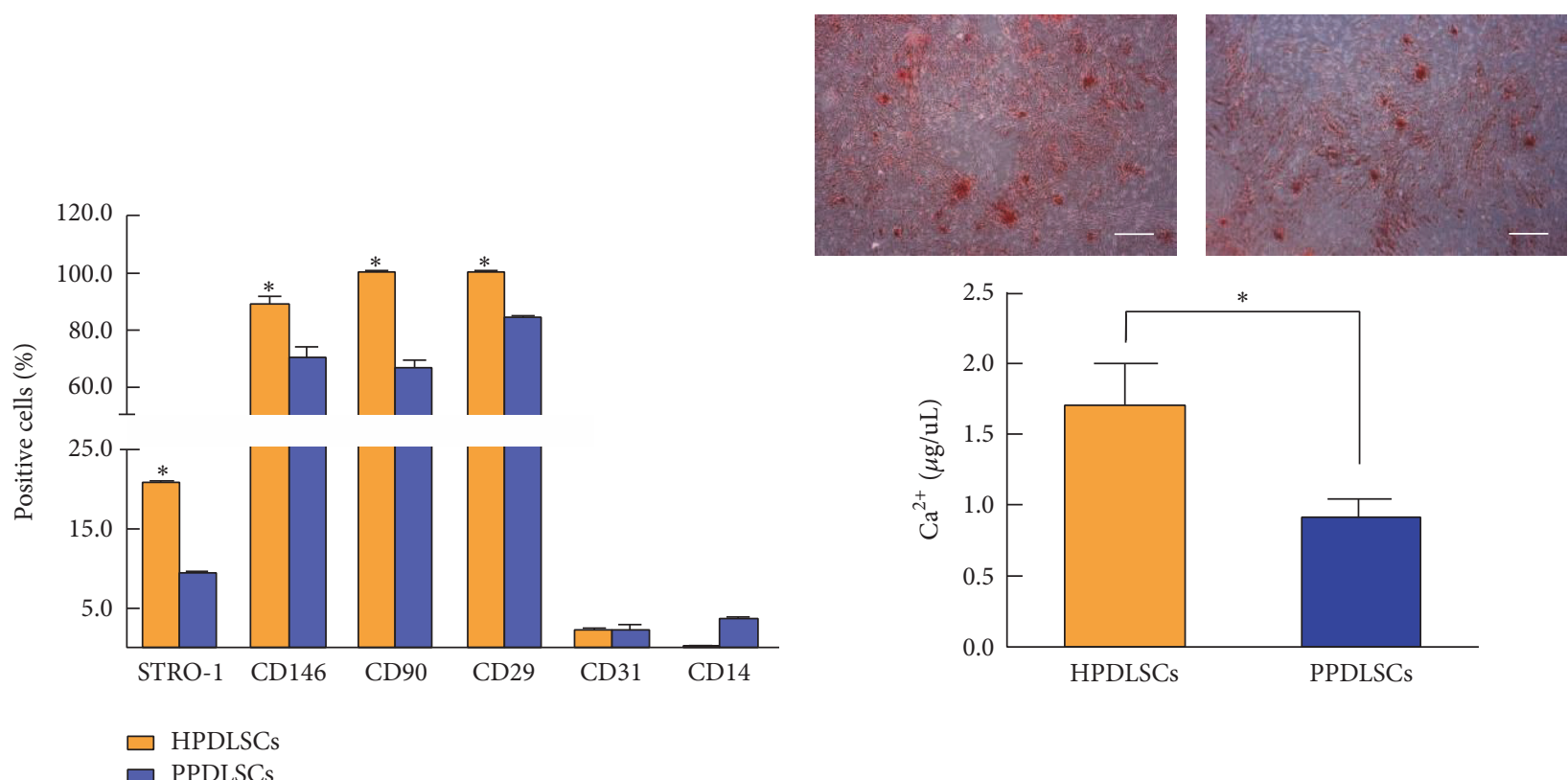

(e) 


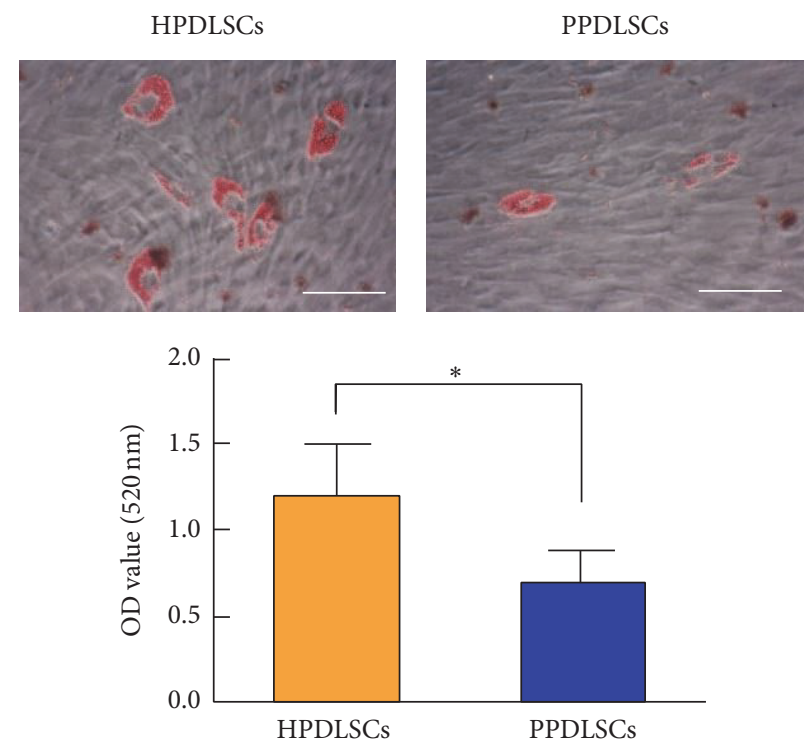

(g)

Figure 1: The cell culture and identification of HPDLSCs and PPDLSCs. (a) Morphologies of HPDLSCs observed by microscopy. (b) Morphologies of PPDLSCs observed by microscopy. (c) Mesenchymal stem cell phenotype examination of HPDLSCs by flow cytometric analysis. (d) Mesenchymal stem cell phenotype examination of PPDLSCs by flow cytometric analysis. (e) Quantitative data for mesenchymal stem cell phenotype examination of HPDLSCs and PPDLSCs. (f) Quantitative analysis of mineralized nodule by calcium level analysis. (g). Quantitative analysis of lipids by isopropanol dissolution and absorbance measurement. ${ }^{*} P<0.05$ versus matched PPDLSCs. Scale bar $=$ $100 \mu \mathrm{m} ; n=6$ in each group. Each experiment was performed three times. The data are presented as the mean \pm standard deviation.

from a healthy microenvironment. Besides, both HPDLSCs and PPDLSCs had the osteogenesis and adipogenesis ability, but due to the impairment of periodontitis, PPDLSCs formed less mineralized nodules and lipid droplets than HPDLSCs $(P<0.05)$ (Figures $1(\mathrm{f})$ and $1(\mathrm{~g}))$.

3.2. Effects of Different Magnitudes of SMS on HPDLSCs and PPDLSCs Proliferation. To evaluate the effects of SMS on HPDLSCs and PPDLSCs proliferation, these two cell populations were exposed to different magnitudes of SMS, and proliferation was examined by cell cycle analysis and MTT assays. Following inflammatory stimulation, PPDLSCs had a higher PI (Figures 2(a) and 2(b)) and greater cell viability (Figure 2(c)) than HPDLSCs in the control groups of stationary cultures $(P<0.05)$. In the SMS groups, all five magnitudes $(6 \%, 8 \%, 10 \%, 12 \%$, and $14 \%$ elongation) increased the proliferation of HPDLSCs, but $12 \%$ SMS had the best effect based on the PI and MTT index $(P<0.05)$; in PPDLSCs, the best SMS value was $8 \%(P<0.05)$, and excessive force decreased both the PI and cell viability (Figures 2(a), 2(b), and 2(c)). These data indicated that SMS differentially influenced the proliferation capacity of HPDLSCs and PPDLSCs. The best SMS value for optimizing proliferation capacity was $12 \%$ for HPDLSCs and $8 \%$ for PPDLSCs.

3.3. Effects of Different Magnitudes of SMS on the Osteogenesis of HPDLSCs and PPDLSCs. To evaluate the effects of different levels of SMS on the osteogenic capacities of HPDLSCs and PPDLSCs, ALP staining, ALP activity assays, Alizarin Red S staining, calcium level analysis, and real-time RT-PCR for osteogenic genes were performed. The results consistently showed that the osteogenic capacity of PPDLSCs was damaged by the inflammatory microenvironment compared with HPDLSCs under conditions with no mechanical stimulation $(P<0.05)$ (Figures 3(a)-3(e)). Exposure to SMS increased ALP activity, mineralized nodule formation, and ALP, Runx2, and OCN gene expression in HPDLSCs in a magnitudedependent manner until the force reached $12 \%$. However, at $14 \%$ SMS, all osteogenic-related indices were decreased $(P<0.05)$ (Figures 3(a)-3(e)). PPDLSCs were more sensitive to SMS than HPDLSCs in terms of osteogenic capacity. The best strain value for PPDLSCs was 8\%, and a stronger stimulus had an adverse effect on osteogenic potential, as evidenced by ALP staining, Alizarin Red S staining, and realtime RT-PCR results $(P<0.05)$ (Figures $3(\mathrm{a})-3(\mathrm{e}))$. These data indicated that the inflammatory microenvironment damaged PPDLSCs function, and the appropriate force level for HPDLSCs was too great for PPDLSCs with regard to osteogenic ability.

3.4. Effects of Different Magnitudes of SMS on the Osteoclastogenesis of HPDLSCs and PPDLSCs. To investigate the effects of SMS on the osteoclastogenesis of HPDLSCs and PPDLSCs, the osteoclastogenesis-related genes RANKL and C-fos were examined by real-time RT-PCR. The levels of these two genes were higher in PPDLSCs than in HPDLSCs in the control groups without SMS, and this result was 
HPDLSCs

Loading
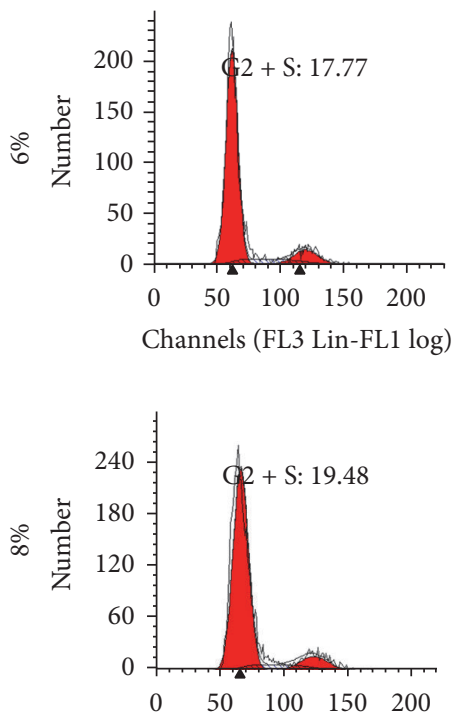

Channels (FL3 Lin-FL1 log)
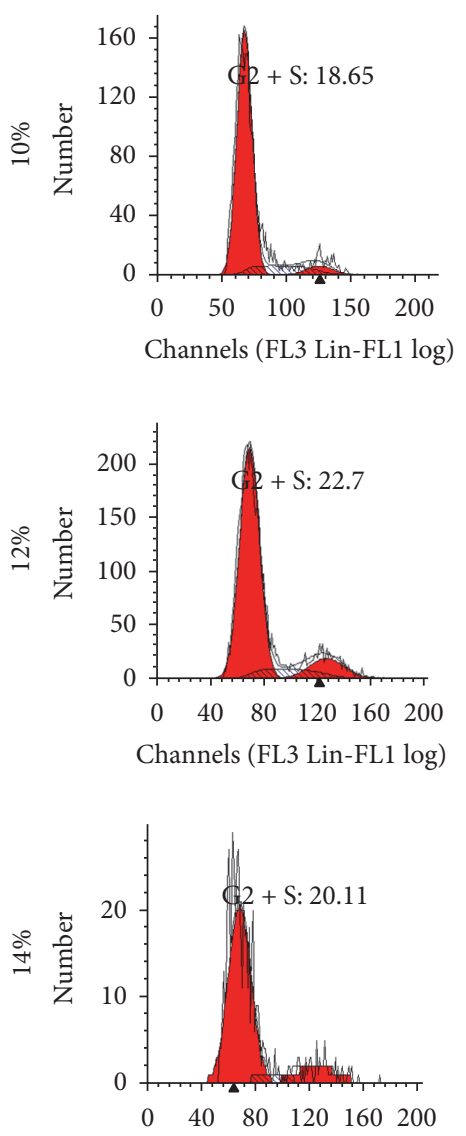

Channels (FL3 Lin-FL1 log)

- Dip G1

- Dip G2

$\square$ Dip S
(+)
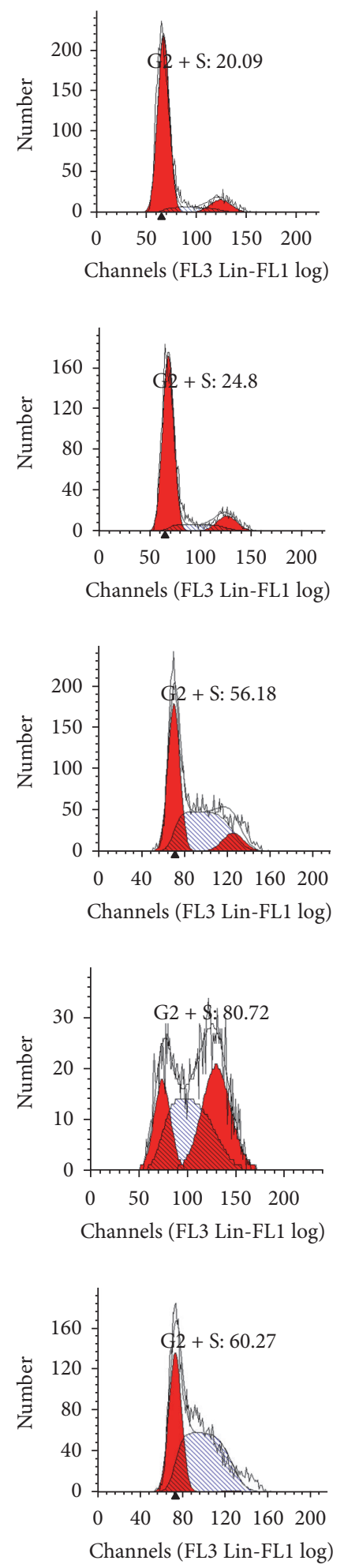

- Dip G1

- Dip G2

๑ Dip S
PPDLSCs
(-)
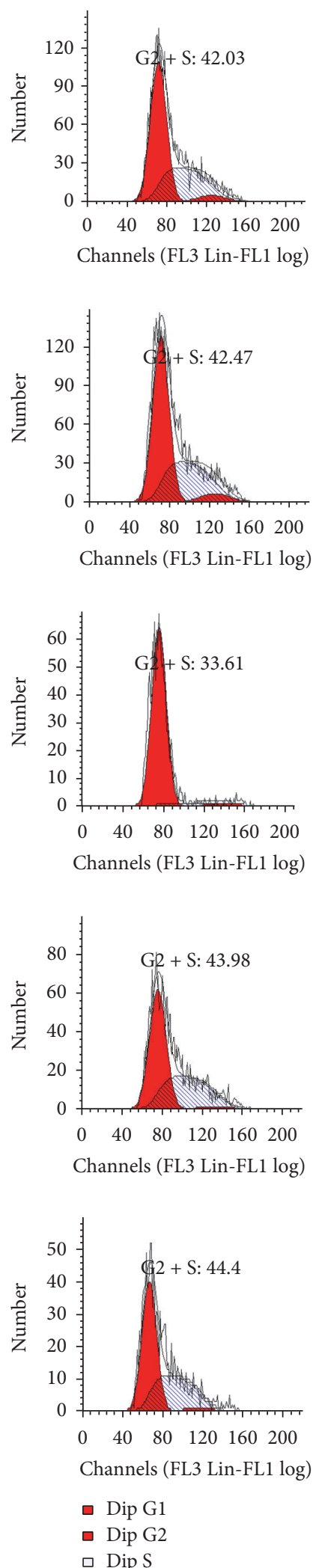

(a)
$(+)$

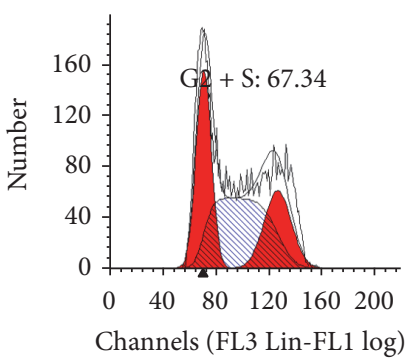

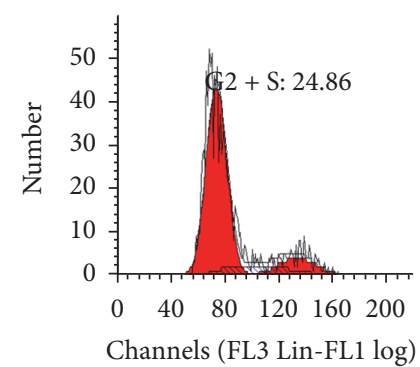
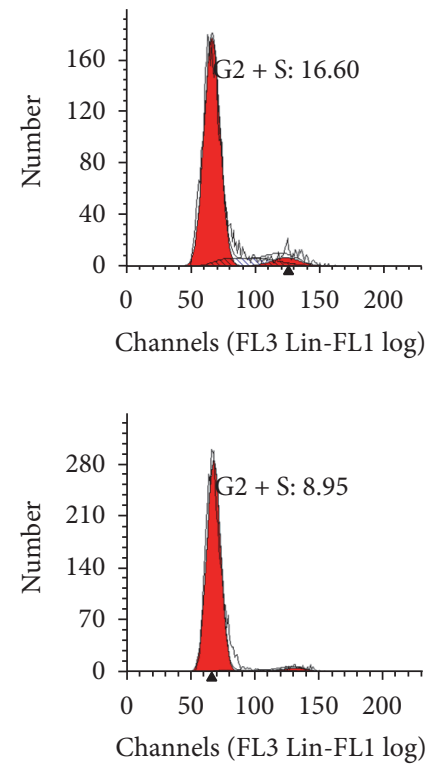

- Dip G1

- Dip G2

$\square$ Dip S

Figure 2: Continued. 


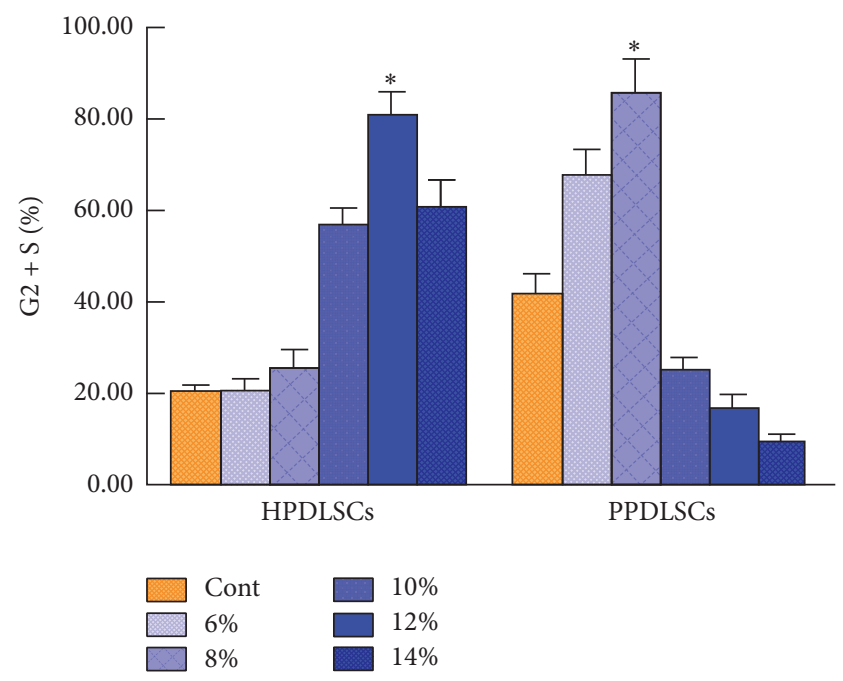

(b)

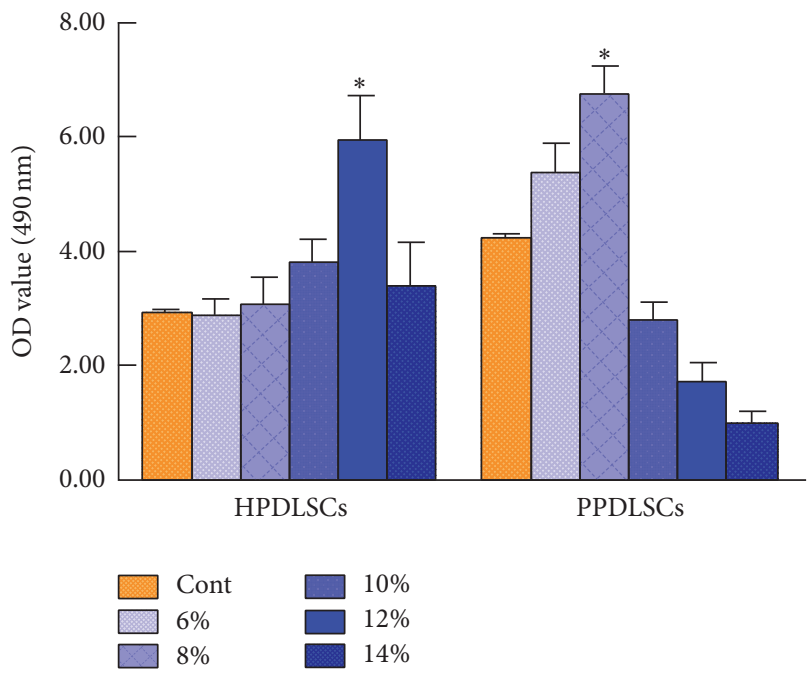

(c)

FIGURE 2: Effects of different magnitudes of SMS on the proliferation of HPDLSCs and PPDLSCs. (a) The percentage of cells in the G2 + S phases was measured by flow cytometry. (b) Quantitative data for the cell cycle analysis. (c) The cell viability was examined by MTT assays. ${ }^{*} P<0.05$ versus the other five groups of HPDLSCs or PPDLSCs; $n=6$ in each group. Each experiment was performed three times. The data are presented as the mean \pm standard deviation.

attributed to inflammatory stimulation $(P<0.05)$ (Figures $4(\mathrm{a})$ and $4(\mathrm{~b})$ ). In the experimental groups, the mRNA levels of RANKL and C-fos demonstrated no significant differences when the strain was less than $12 \%$ in HPDLSCs and less than $8 \%$ in PPDLSCs. However, when the strain was higher than $12 \%$ in HPDLSCs, the mRNA levels of RANKL and C-fos were obviously increased, and PPDLSCs showed a similar tendency when the strain was higher than $8 \%$ $(P<0.05)$ (Figures 4(a) and 4(b)). These data indicated that, in HPDLSCs, an SMS higher than 12\% activated the expression of osteoclastogenic genes, while PPDLSCs were more sensitive to mechanical stimulation; an SMS higher than $8 \%$ activated osteoclastogenesis in PPDLSCs.

\subsection{Effects of Different Magnitudes of SMS on the Inflamma-} tory Response of HPDLSCs and PPDLSCs. To identify the effects of SMS on the inflammatory response of HPDLSCs and PPDLSCs, the secretion of inflammatory cytokines, including IL-6, IL-8, IL-1 $\beta$, and TNF- $\alpha$, was detected using ELISA assays. PPDLSCs secreted more inflammatory cytokines than HPDLSCs under conditions with no SMS $(P<0.05)$ (Figures 5(a)-5(d)). When SMS was applied, the secretion of IL-1 $\beta$ and TNF- $\alpha$ by both HPDLSCs and PPDLSCs was slightly increased, but different levels of SMS made almost no difference in each group $(P>0.05)$ (Figures $5(\mathrm{a})$ and 5(b)). Nevertheless, the levels of IL-6 and IL- 8 were significantly upregulated by approximately hundredfold. In identifying the minimal inflammatory response, we found that an SMS less than 12\% in HPDLSCs and less than $8 \%$ in PPDLSCs induced slight production of IL- 6 and IL-8, which was beneficial for tissue regeneration $(P<0.05)$ (Figures 5(c) and 5(d)).

\section{Discussion}

During orthodontic treatment, the transmission of mechanical force to alveolar bone is mediated by the response of the PDL, leading to adaptation of periodontal tissues to the mechanical force [22]. This response primarily depends on cells in the PDL. A study by Theilig et al. [23] showed that cell attachment and expression of the ECM glycoprotein tenascin by human periodontal ligament cells (HPDLCs) were obviously changed after centrifugation. The literature [14] has also documented that low-magnitude and highfrequency mechanical vibration increase ALP activity and OCN expression, ultimately promoting the osteogenic differentiation of PDLSCs in a frequency-dependent manner. However, excessive orthodontic force may be an important cofactor for periodontium damage and the pathogenesis of periodontal diseases. Kim et al. [15] confirmed that periodontal ligament cells (PDLCs) continuously produce RANKL, which provides a crucial signal for osteoclast formation, when subjected to a sustaining orthodontic force. Yamamoto et al. [7] found that PDLCs produced many types of cytokines in response to bacterial stimulation, and in the presence of local inflammation in periodontal tissues, mechanical stress aggravated the inflammatory response and led to alveolar bone resorption.

In our previous study, we found that PDLSCs function was impaired in a periodontitis microenvironment, which may contribute to the deterioration of the regeneration ability of the periodontium [16]. However, the precise mechanisms underlying the different responses of healthy and inflammatory periodontium to mechanical force remained unclear. Furthermore, whether cells derived from tissues in these distinct microenvironments, including HPDLSCs and 


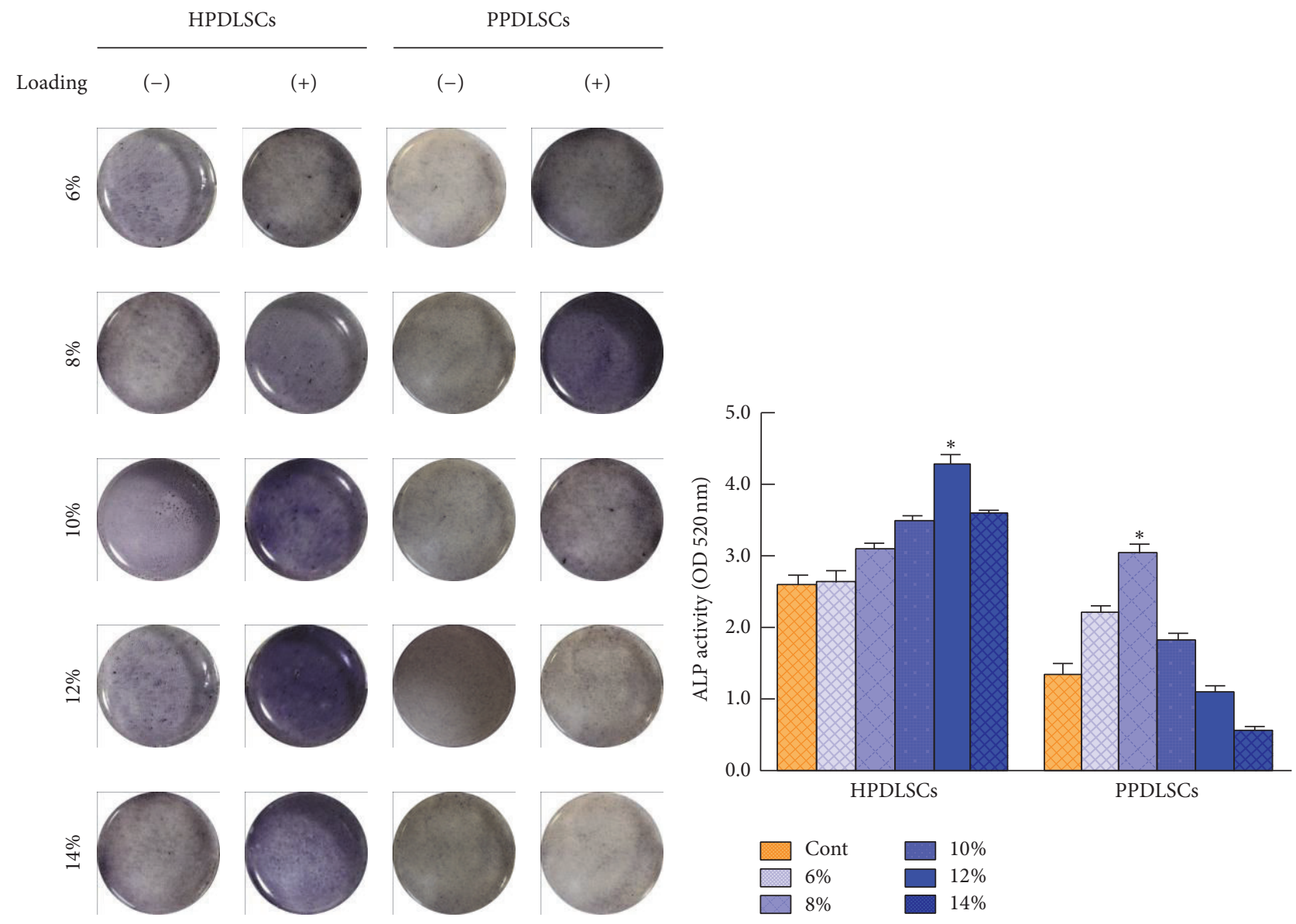

(a)

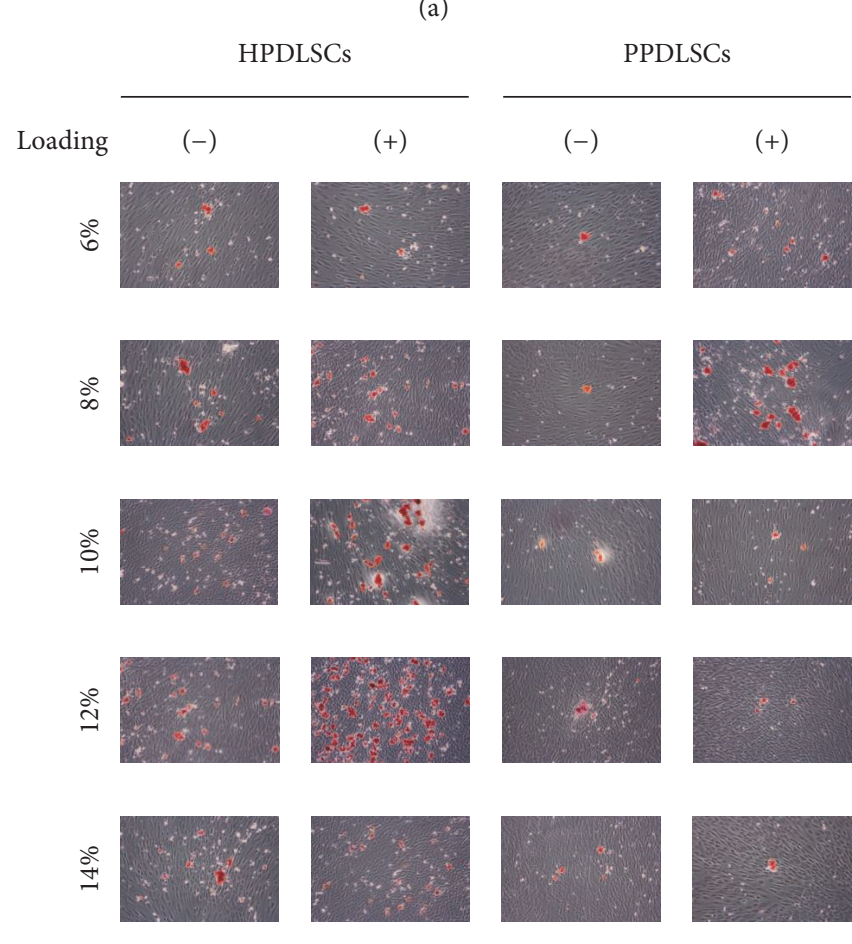

(b)

(c)

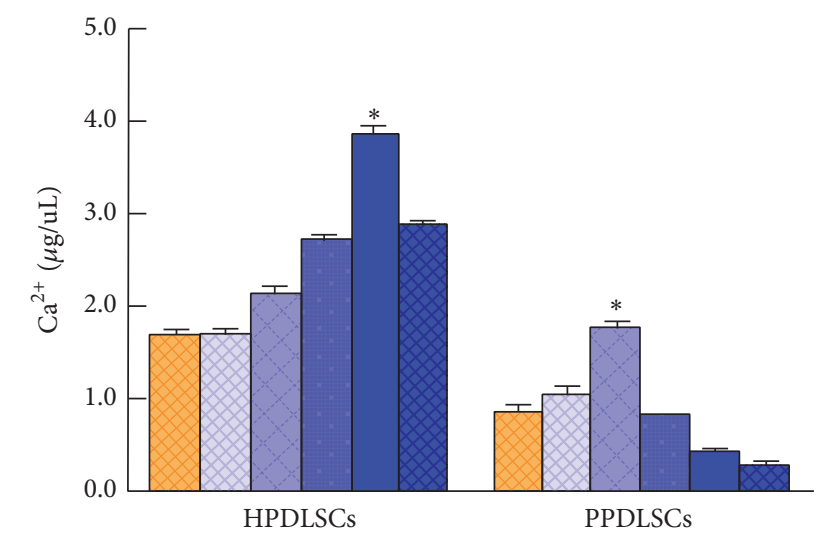

FIGURE 3: Continued. 

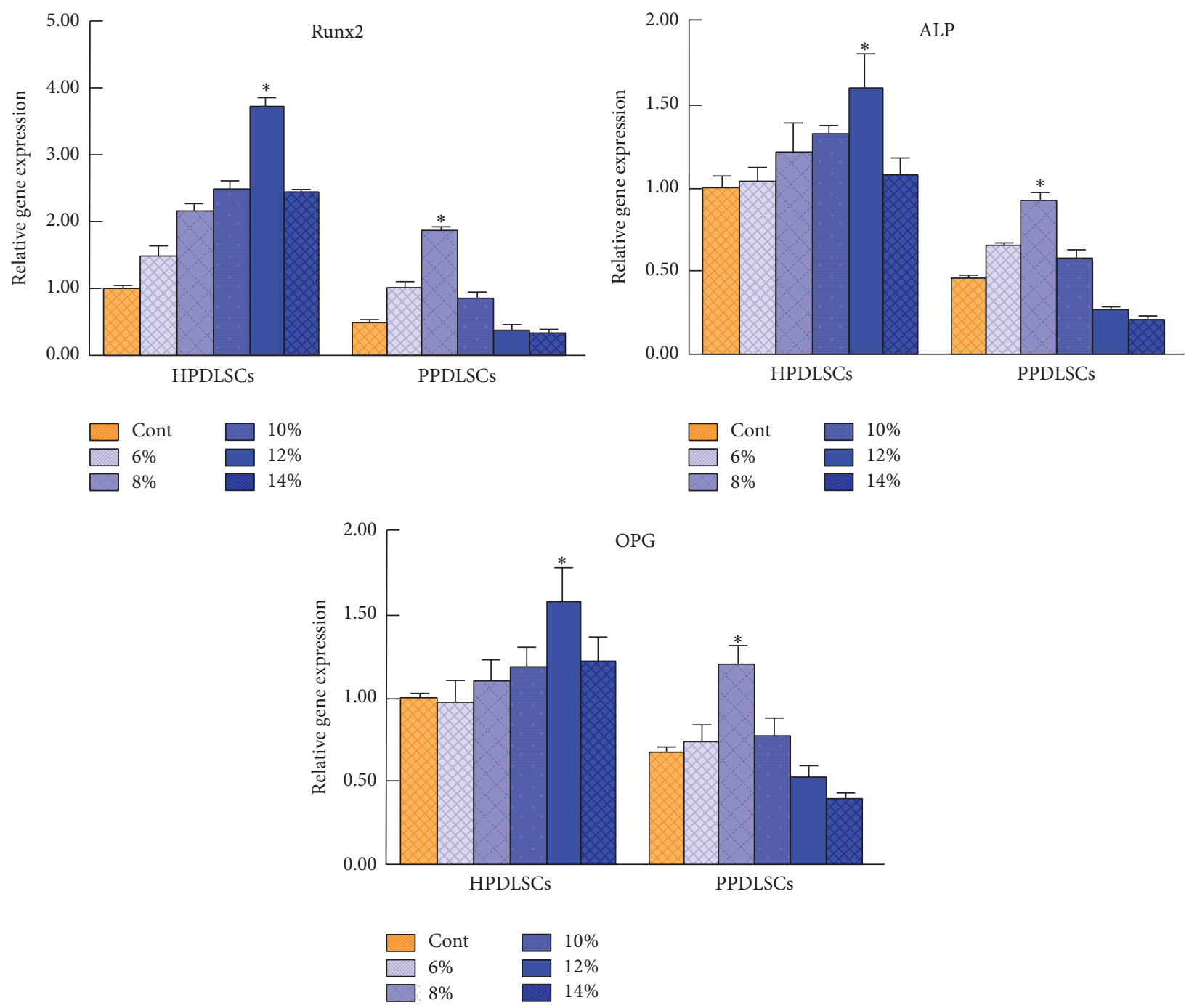

(e)

FIGURE 3: Effects of different magnitudes of SMS on the osteogenesis of HPDLSCs and PPDLSCs. (a) ALP activity was detected by ALP staining. (b) Quantitative analysis of ALP activity. (c) Osteogenic differentiation was determined by Alizarin Red S staining. (d) Quantitative analysis of calcium concentration was performed by calcium level analysis. (e) The expression levels of the osteogenic genes Runx2, ALP, and OPG were measured by real-time PCR. ${ }^{*} P<0.05$ versus the other five groups of HPDLSCs or PPDLSCs; $n=6$ in each group. Each experiment was performed three times. The data are presented as the mean \pm standard deviation.

PPDLSCs, reacted differently to a mechanical stimulus was unknown. The purpose of this study was to evaluate whether SMS exerted different effects on HPDLSCs and PPDLSCs and to determine the optimal SMS value for periodontal remodeling and regeneration by HPDLSCs and PPDLSCs.

We conducted this study by establishing a cell-loading system using a Flexcell FX-4000T device. A numerical analysis of tooth mobility by Natali et al. [24] has demonstrated that maximal PDL strains for translational movements of a human tooth under physiological loading conditions are in the vicinity of $8 \%-25 \%$, and the value of $12 \%$ correlates well with the strain conditions at the midroot. Besides, previous studies have shown that the mechanical strain of $12 \%$ along with the frequency of $0.1 \mathrm{~Hz}$ was the commonly used force character for periodontal-related cells $[19,25,26]$. Since we assumed that PPDLSCs were more sensitive and thus the optimal value for PPDLSCs should be lower than that for HPDLSCs, we analyzed a range of magnitudes from $6 \%$ to $14 \%$ with $0.1 \mathrm{~Hz}$ frequency to address the purpose of our study. The loading time of mechanical force is important for tissue reaction. Periodontal tissue will remodel when the force is lasting for enough time. When the mechanical strain lasted for $1 \mathrm{~h}$, the viability of PDLCs presented no changes [7]; while the orthodontic force lasted for $12 \mathrm{~h}$, genes and proteins in periodontal tissue began to activate and change $[27,28]$. Therefore, we chose $12 \mathrm{~h}$ as the loading time in the present study. PPDLSCs were harvested from periodontitis tissues when the periodontitis was in a static phase. Although the PPDLSCs were separated from the inflammatory microenvironment, the functional deficit 


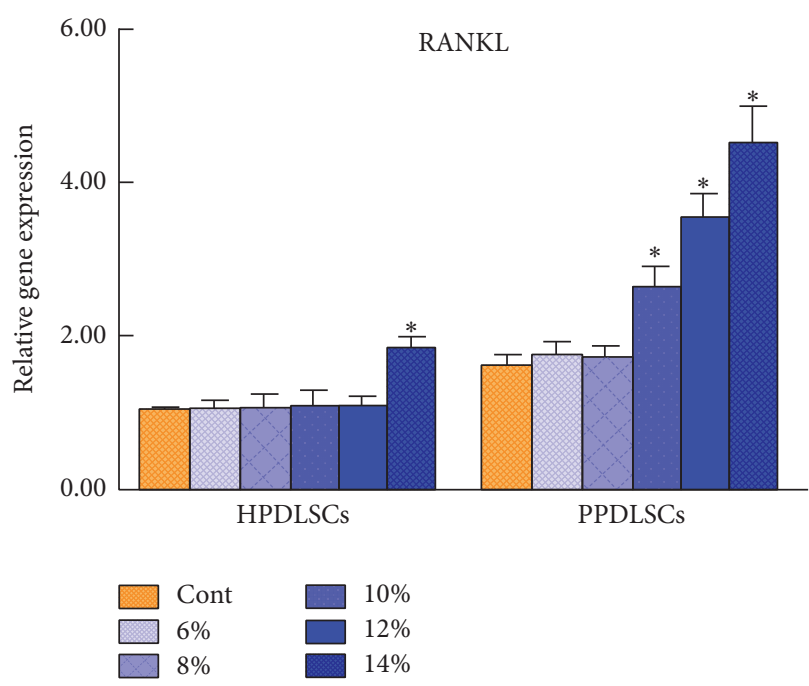

(a)

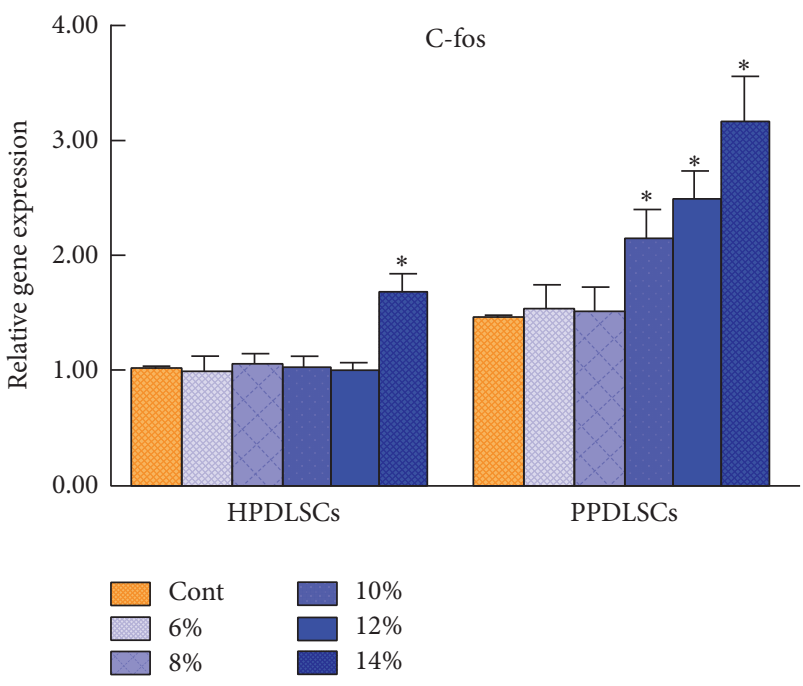

(b)

FIgURE 4: Effects of different magnitudes of SMS on the osteoclastogenesis of HPDLSCs and PPDLSCs. (a) The expression levels of the osteoclastogenic gene RANKL by real-time PCR. (b) The expression levels of the osteoclastogenic gene C-fos by real-time PCR. ${ }^{*} P<0.05$ versus the control group of HPDLSCs or PPDLSCs; $n=6$ in each group. Each experiment was performed three times. The data are presented as the mean \pm standard deviation.

could not be recovered during ex vivo culture and expansion, according to our current findings and previous studies by our group [16, 29]. Because the periodontitis was chronic in the patients, and the condition was persistent for a long time, we assumed that epigenetic modification could play a critical role in maintaining the functional deficit [30, 31]. It was reported that epigenetics is closely related to inflammation, including in periodontitis $[32,33]$. For example, DNA methylation and histone modification have been shown to be critical in the regulation of inflammatory genes [34], and noncoding RNA regulates the expression of target genes at the posttranscriptional level to alter PDLSCs function by epigenetic mechanisms [35]. In our future studies, the specific mechanisms by which epigenetics affect cell function and the response of PPDLSCs to SMS will be considered.

Proliferation is necessary for tissue homeostasis and regeneration by MSCs. In our study, cell cycle and MTT assays were performed to detect the proliferation abilities of HPDLSCs and PPDLSCs. PPDLSCs had a higher proliferation ability than HPDLSCs, which was consistent with previous studies $[9,16]$. Moreover, the two populations of PDLSCs responded differently to SMS. The best force value for proliferation potential was $12 \%$ for HPDLSCs, while for PPDLSCs, an SMS value higher than $8 \%$ suppressed cell viability and decreased the PI. These SMS values indicated that PPDLSCs were more sensitive and less tolerant to SMS than HPDLSCs in terms of proliferation ability.

The process of orthodontic tooth movement should be performed in a manner that balances osteogenesis and osteoclastogenesis, and this balance can be disrupted if the periodontal tissues are in an inflammatory environment [36]. A series of experiments demonstrated that an SMS of $6 \%$ to $14 \%$ exerted distinct effects on HPDLSCs and PPDLSCs.
Specifically, the osteogenic ability of HPDLSCs was upregulated in a magnitude-dependent manner when the cells were exposed to an SMS less than 12\%, and the osteoclastogenic genes were barely activated. When the SMS was increased to $14 \%$, the osteogenic ability of HPDLSCs decreased, and the expression of RANKL and C-fos significantly increased, indicating that the balance between osteogenesis and osteoclastogenesis was disrupted. For PPDLSCs, any SMS higher than $8 \%$ suppressed osteogenic ability and activated osteoclastogenesis.

Mechanical stimuli can regulate MSCs [37] and PDLSCs [14] functions, including proliferation, differentiation, and cytokine secretion [38]. Excessive inflammatory cytokine secretion can result in periodontal damage; therefore, it is crucial to minimize the production of inflammatory cytokines in the presence of SMS. In our study, the concentrations of IL-1 $\beta$ and TNF- $\alpha$ in supernatants from both HPDLSCs and PPDLSCs increased slightly under different levels of SMS, but the trend was not significant. Exposure to SMS increased IL-6 and IL-8 secretion to a very high level. We speculated that the mechanical stimulus promoted IL- 6 and IL-8 secretion, which might participate in the inflammatory response underlying periodontal remodeling. These findings also suggested that PPDLSCs should receive less SMS than HPDLSCs to minimize inflammatory injury.

\section{Conclusions}

In conclusion, excessive mechanical force and periodontitisrelated inflammation led to increased destruction of periodontal tissue, as previously reported [39-42]. Our results demonstrated that HPDLSCs and PPDLSCs responded differently to SMS. Specifically, PPDLSCs were more sensitive 


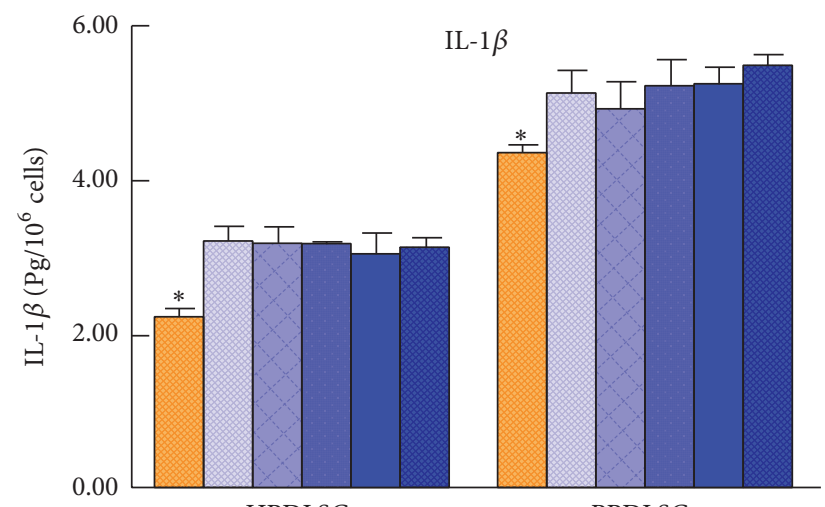

HPDLSCs

PPDLSCs

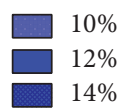

(a)

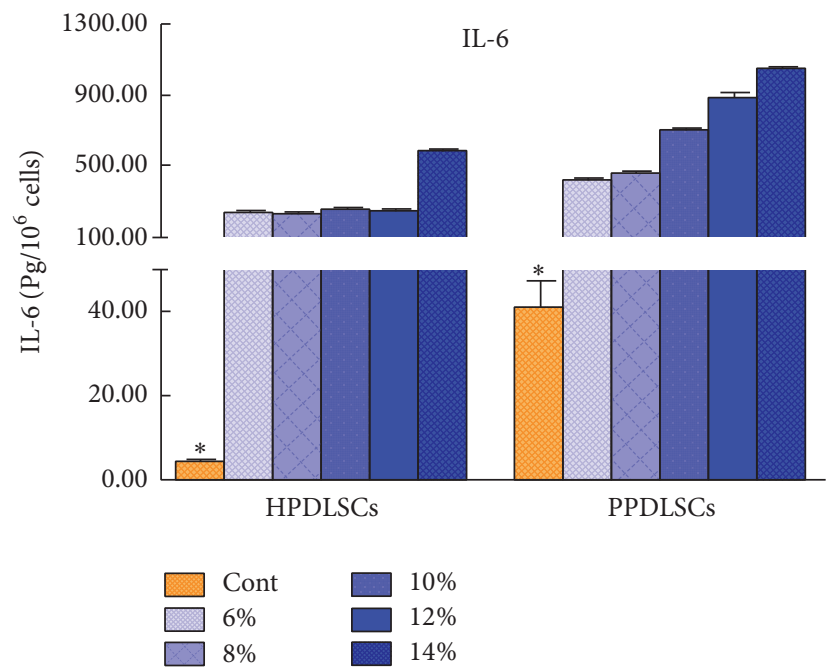

(c)

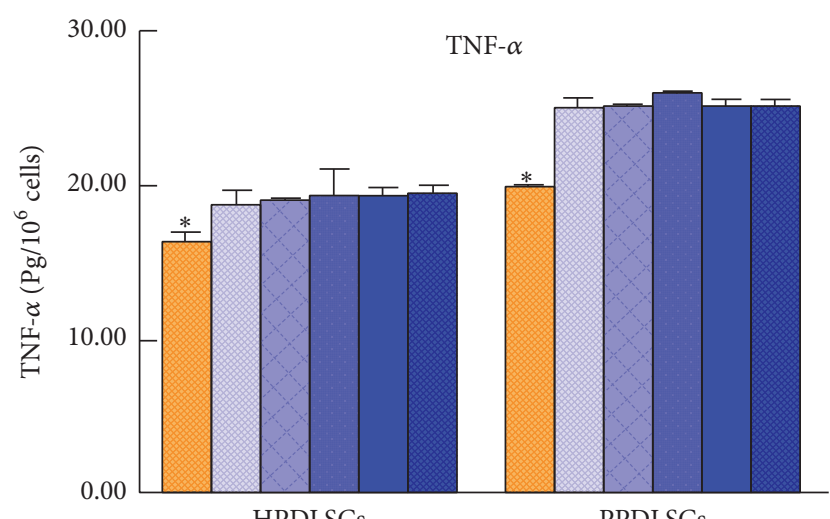

HPDLSCs

PPDLSCs

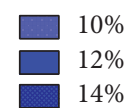

(b)

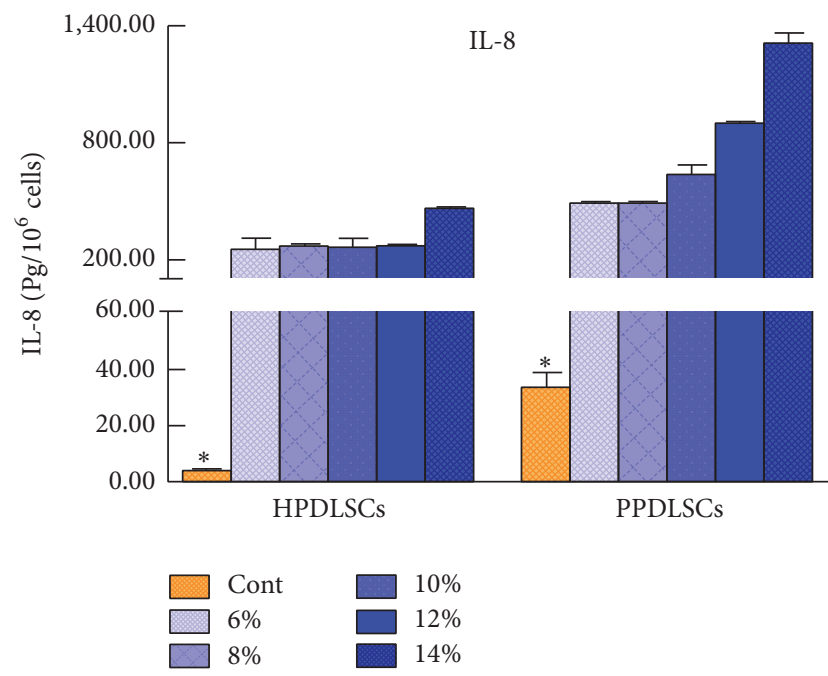

(d)

FIGURE 5: Effects of different magnitudes of SMS on the inflammatory responses in HPDLSCs and PPDLSCs. (a) The protein levels of the inflammatory cytokine IL-1 $\beta$ were determined by ELISA. (b) The protein levels of the inflammatory cytokine TNF- $\alpha$ were determined by ELISA. (c) The protein levels of the inflammatory cytokine IL-6 were determined by ELISA. (d) The protein levels of the inflammatory cytokine IL- 8 were determined by ELISA. ${ }^{*} P<0.05$ versus the other five SMS loading groups of HPDLSCs or PPDLSCs; $n=6$ in each group. Each experiment was performed three times. The data are presented as the mean \pm standard deviation.

and less tolerant to SMS; an SMS of $8 \%$ was the best in terms of the regeneration ability of PPDLSCs, while the best magnitude for HPDLSCs was $12 \%$. In the periodontitis microenvironment, excessive mechanical loading can lead to decreased proliferation and osteogenic capacity, the activation of osteoclastogenesis, and increased secretion of inflammatory factors by PPDLSCs, all of which are detrimental for periodontal remodeling in the presence of orthodontic force. These results suggest that an orthodontist should perform light and moderate force to the teeth during orthodontic treatments for patients with periodontitis. However, this study was performed at the cytological level; in future studies, we will determine how to transform the optimal SMS from the cellular level to the tissue level and ascertain the precise mechanisms underlying the differential responses of HPDLSCs and PPDLSCs to SMS.

\section{Competing Interests}

The authors declare no potential competing interests.

\section{Acknowledgments}

This work was supported by the National Natural Science Foundation of China (nos. 81500851 and 81271176), the Natural Science Foundation of Shaanxi Province (no. 2016JQ8013), and the Academic Conference Funding of the School of Stomatology, FMMU (no. 2014JS-01). 


\section{References}

[1] G. E. Wise and G. J. King, "Mechanisms of tooth eruption and orthodontic tooth movement," Journal of Dental Research, vol. 87, no. 5, pp. 414-434, 2008.

[2] R. S. Masella and M. Meister, "Current concepts in the biology of orthodontic tooth movement," American Journal of Orthodontics and Dentofacial Orthopedics, vol. 129, no. 4, pp. 458-468, 2006.

[3] A. S. Narayanan and R. C. Page, "Connective tissues of the periodontium: a summary of current work," Collagen and Related Research, vol. 3, no. 1, pp. 33-64, 1983.

[4] S. M. Gondivkar, R. S. Gondivkar, A. R. Gadbail, R. Chole, M. Mankar, and M. Yuwanati, "Chronic periodontitis and the risk of head and neck squamous cell carcinoma: facts and figures," Experimental Oncology, vol. 35, no. 3, pp. 163-167, 2013.

[5] K. Kobayashi, N. Takahashi, E. Jimi et al., "Tumor necrosis factor $\alpha$ stimulates osteoclast differentiation by a mechanism independent of the ODF/RANKL-RANK interaction," The Journal of Experimental Medicine, vol. 191, no. 2, pp. 275-286, 2000.

[6] Y.-S. Kim, Y.-M. Lee, J.-S. Park, S.-K. Lee, and E.-C. Kim, "SIRT1 modulates high-mobility group box 1-induced osteoclastogenic cytokines in human periodontal ligament cells," Journal of Cellular Biochemistry, vol. 111, no. 5, pp. 1310-1320, 2010.

[7] T. Yamamoto, M. Kita, K. Yamamoto, Y. Akamatsu, F. Oseko, and N. Kanamura, "Mechanical stress enhances production of cytokines in human periodontal ligament cells induced by Porphyromonas gingivalis," Archives of Oral Biology, vol. 56, no. 3, pp. 251-257, 2011.

[8] G. T. Huang, S. Gronthos, and S. Shi, "Mesenchymal stem cells derived from dental tissues vs. those from other sources: their biology and role in regenerative medicine," Journalof Dental Research, vol. 88, no. 9, pp. 792-806, 2009.

[9] N. Liu, S. Shi, M. Deng et al., "High levels of $\beta$-catenin signaling reduce osteogenic differentiation of stem cells in inflammatory microenvironments through inhibition of the noncanonical Wnt pathway," Journal of Bone and Mineral Research, vol. 26, no. 9, pp. 2082-2095, 2011.

[10] V. Krishnan and Z. Davidovitch, "Cellular, molecular, and tissue-level reactions to orthodontic force," American Journal of Orthodontics and Dentofacial Orthopedics, vol. 129, no. 4, pp. 469.el-469.e32, 2006.

[11] B. M. Seo, M. Miura, S. Gronthos et al., "Investigation of multipotent postnatal stem cells from human periodontal ligament," The Lancet, vol. 364, no. 9429, pp. 149-155, 2004.

[12] H. F. Rios, Z. Lin, B. Oh, C. H. Park, and W. V. Giannobile, "Cell- and gene-based therapeutic strategies for periodontal regenerative medicine," Journal of Periodontology, vol. 82, no. 9, pp. 1223-1237, 2011.

[13] F.-M. Chen, H.-H. Sun, H. Lu, and Q. Yu, "Stem cell-delivery therapeutics for periodontal tissue regeneration," Biomaterials, vol. 33, no. 27, pp. 6320-6344, 2012.

[14] C. Zhang, J. Li, L. Zhang et al., "Effects of mechanical vibration on proliferation and osteogenic differentiation of human periodontal ligament stem cells," Archives of Oral Biology, vol. 57, no. 10, pp. 1395-1407, 2012.

[15] T. Kim, A. Handa, J. Iida, and S. Yoshida, "RANKL expression in rat periodontal ligament subjected to a continuous orthodontic force," Archives of Oral Biology, vol. 52, no. 3, pp. 244-250, 2007.

[16] J. Liu, L. Wang, W. Liu, Q. Li, Z. Jin, and Y. Jin, "Dental follicle cells rescue the regenerative capacity of periodontal ligament stem cells in an inflammatory microenvironment," PLoS One, vol. 9, no. 9, p. e108752, 2014.

[17] W. Zheng, S. Wang, D. Ma, L. Tang, Y. Duan, and Y. Jin, "Loss of proliferation and differentiation capacity of aged human periodontal ligament stem cells and rejuvenation by exposure to the young extrinsic environment," Tissue engineering. Part A, vol. 15, no. 9, pp. 2363-2371, 2009.

[18] J. Zhang, Y. An, L.-N. Gao, Y.-J. Zhang, Y. Jin, and F.-M. Chen, "The effect of aging on the pluripotential capacity and regenerative potential of human periodontal ligament stem cells," Biomaterials, vol. 33, no. 29, pp. 6974-6986, 2012.

[19] M. Liu, J. Dai, Y. Lin et al., "Effect of the cyclic stretch on the expression of osteogenesis genes in human periodontal ligament cells," Gene, vol. 491, no. 2, pp. 187-193, 2012.

[20] L.-N. Gao, Y. An, M. Lei et al., "The effect of the coumarinlike derivative osthole on the osteogenic properties of human periodontal ligament and jaw bone marrow mesenchymal stem cell sheets," Biomaterials, vol. 34, no. 38, pp. 9937-9951, 2013.

[21] W. Liu, A. Konermann, T. Guo, A. Jäger, L. Zhang, and Y. Jin, "Canonical Wnt signaling differently modulates osteogenic differentiation of mesenchymal stem cells derived from bone marrow and from periodontal ligament under inflammatory conditions," Biochimica et Biophysica Acta, vol. 1840, no. 3, pp. 1125-1134, 2014.

[22] P. Lekic and C. A. G. Mcculloch, "Periodontal ligament cell populations: the central role of fibroblasts in creating a unique tissue," The Anatomical Record, vol. 245, no. 2, pp. 327-341, 1996.

[23] C. Theilig, A. Bernd, G. Leyhausen, R. Kaufmann, and W. Geurtsen, "Effects of mechanical force on primary human fibroblasts derived from the gingiva and the periodontal ligament," Journal of Dental Research, vol. 80, no. 8, pp. 1777-1780, 2001.

[24] A. N. Natali, P. G. Pavan, and C. Scarpa, "Numerical analysis of tooth mobility: formulation of a non-linear constitutive law for the periodontal ligament," Dental Materials, vol. 20, no. 7, pp. 623-629, 2004.

[25] V. Terraciano, N. Hwang, L. Moroni et al., "Differential response of adult and embryonic mesenchymal progenitor cells to mechanical compression in hydrogels," Stem Cells, vol. 25, no. 11, pp. 2730-2738, 2007.

[26] C. Jacobs, S. Grimm, T. Ziebart, C. Walter, and H. Wehrbein, "Osteogenic differentiation of periodontal fibroblasts is dependent on the strength of mechanical strain," Archives of Oral Biology, vol. 58, no. 7, pp. 896-904, 2013.

[27] G. A. Rodan and T. J. Martin, "Role of osteoblasts in hormonal control of bone resorption-A hypothesis," Calcified Tissue International, vol. 33, no. 1, pp. 349-351, 1981.

[28] W. E. Roberts and E. R. Morey, "Proliferation and differentiation sequence of osteoblast histogenesis under physiological conditions in rat periodontal ligament," American Journal of Anatomy, vol. 174, no. 2, pp. 105-118, 1985.

[29] X. Kong, Y. Liu, R. Ye et al., "GSK3 $\beta$ is a checkpoint for TNF- $\alpha$ mediated impaired osteogenic differentiation of mesenchymal stem cells in inflammatory microenvironments," Biochimica et Biophysica Acta-General Subjects, vol. 1830, no. 11, pp. 51195129, 2013.

[30] Y.-H. Jiang, J. Bressler, and A. L. Beaudet, "Epigenetics and human disease," Annual Review of Genomics and Human Genetics, vol. 5, pp. 479-510, 2004.

[31] A. Bird, "Perceptions of epigenetics," Nature, vol. 447, no. 7143, pp. 396-398, 2007. 
[32] D. Bayarsaihan, "Epigenetic mechanisms in inflammation," Journal of Dental Research, vol. 90, no. 1, pp. 9-17, 2011.

[33] M. D. Martins, Y. Jiao, L. Larsson et al., "Epigenetic modifications of histones in periodontal disease," Journal of Dental Research, vol. 95, no. 2, pp. 215-222, 2016.

[34] R. Medzhitov and T. Horng, "Transcriptional control of the inflammatory response," Nature Reviews Immunology, vol. 9, no. 10, pp. 692-703, 2009.

[35] Y. Liu, W. Liu, C. Hu et al., "MiR-17 modulates osteogenic differentiation through a coherent feed-forward loop in mesenchymal stem cells isolated from periodontal ligaments of patients with periodontitis," Stem Cells, vol. 29, no. 11, pp. 18041816, 2011.

[36] H. Hazan-Molina, L. Levin, S. Einy, and D. Aizenbud, "Aggressive periodontitis diagnosed during or before orthodontic treatment," Acta Odontologica Scandinavica, vol. 71, no. 5, pp. 1023-1031, 2013.

[37] J. Hao, Y. Zhang, D. Jing et al., "Mechanobiology of mesenchymal stem cells: perspective into mechanical induction of MSC fate," Acta Biomaterialia, vol. 20, pp. 1-9, 2015.

[38] S.-B. Zhou, J. Wang, C.-A. Chiang, L.-L. Sheng, and Q.-F. $\mathrm{Li}$, "Mechanical stretch upregulates SDF- $1 \alpha$ in skin tissue and induces migration of circulating bone marrow-derived stem cells into the expanded skin," Stem Cells, vol. 31, no. 12, pp. 27032713, 2013.

[39] L. J. Jin and C. F. Cao, "Clinical diagnosis of trauma from occlusion and its relation with severity of periodontitis," Journal of Clinical Periodontology, vol. 19, no. 2, pp. 92-97, 1992.

[40] N. Shimizu, M. Yamaguchi, K. Uesu, T. Goseki, and Y. Abiko, "Stimulation of prostaglandin E2 and interleukin-1 $\beta$ production from old rat periodontal ligament cells subjected to mechanical stress," The Journals of Gerontology, Series A: Biological Sciences and Medical Sciences, vol. 55, no. 10, pp. B489-B495, 2000.

[41] P. Long, J. Hu, N. Piesco, M. Buckley, and S. Agarwal, "Low magnitude of tensile strain inhibits IL- $1 \beta$-dependent induction of pro-inflammatory cytokines and induces synthesis of IL-10 in human periodontal ligament cells in vitro," Journal of Dental Research, vol. 80, no. 5, pp. 1416-1420, 2001.

[42] M. Morita, K. Nishi, T. Kimura et al., "Correlation between periodontal status and biting ability in Chinese adult population," Journal of Oral Rehabilitation, vol. 30, no. 3, pp. 260-264, 2003. 

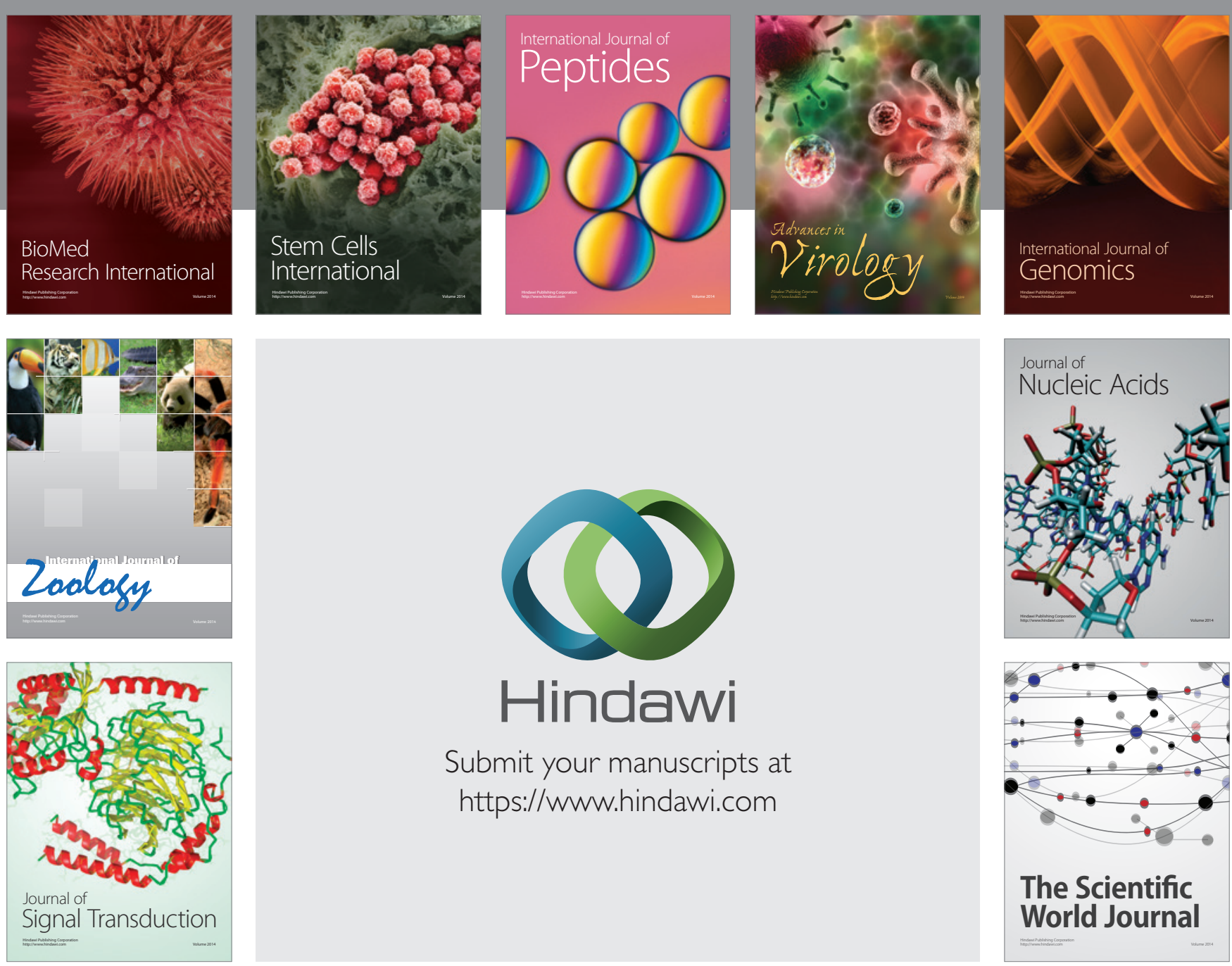

Submit your manuscripts at

https://www.hindawi.com
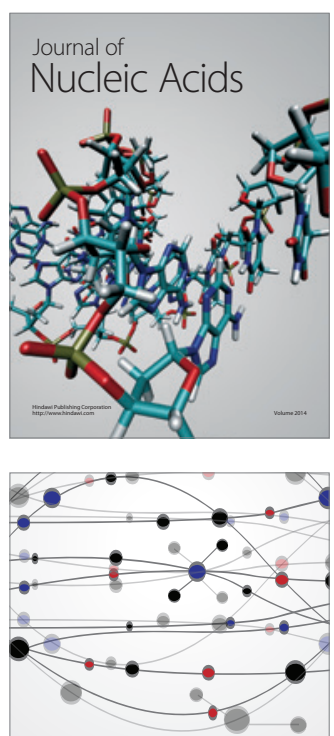

The Scientific World Journal
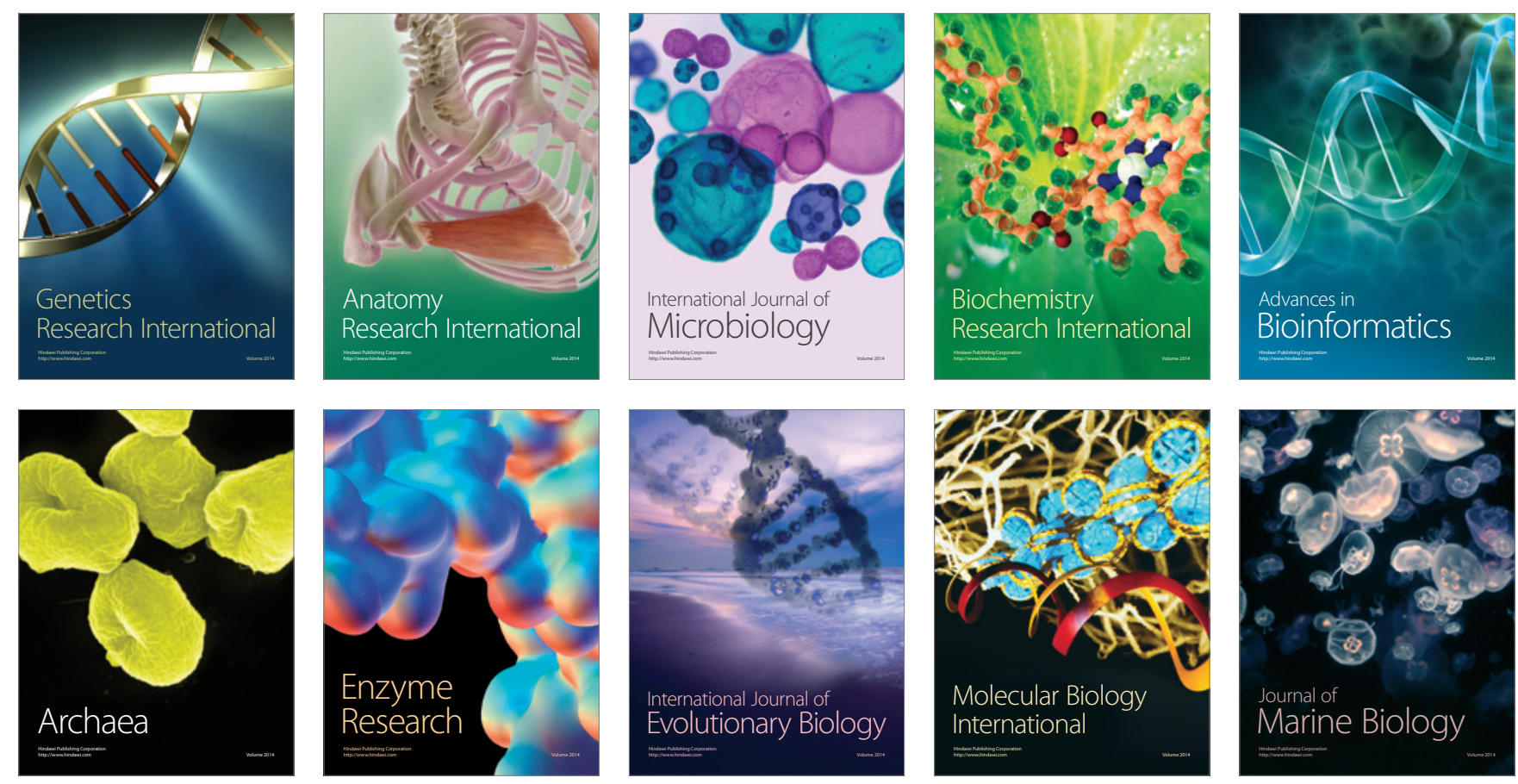